\title{
Characterization of vorticity in pygmy resonances and soft-dipole modes with two-nucleon transfer reactions
}

\author{
R.A. Broglia ${ }^{1,2}$, F. Barranco ${ }^{3}$, G. Potel ${ }^{4}$, and E. Vigezzi ${ }^{5}$ \\ 1 The Niels Bohr Institute, University of Copenhagen, DK-2100 Copenhagen, Blegdamsvej 17, Denmark \\ 2 Dipartimento di Fisica, Università degli Studi Milano, Via Celoria 16, I-20133 Milano, Italy \\ 3 Departamento de Fìsica Aplicada III, Escuela Superior de Ingenieros, Universidad de Sevilla, Camino de los Descubrimientos, \\ Sevilla, Spain \\ 4 National Superconducting Cyclotron Laboratory, Michigan State University, East Lansing, Michigan 48824, USA \\ 5 INFN Sezione di Milano, Via Celoria 16, I-20133 Milano, Italy
}

the date of receipt and acceptance should be inserted later

\begin{abstract}
The properties of the two-quasiparticle-like soft E1-modes and PDR have been and are systematically studied with the help of inelastic and electromagnetic experiments which essentially probe the particle-hole components of these vibrations. It is shown that further insight in their characterisation can be achieved with the help of two-nucleon transfer reactions, in particular concerning the particle-particle components of the modes, in terms of absolute differential cross sections which take properly into account successive and simultaneous transfer mechanisms corrected for non-orthogonality, able to reproduce the experimental findings at the $10 \%$ level. The process ${ }^{9} \mathrm{Li}(t, p){ }^{11} \mathrm{Li}\left(1^{-}\right)$is discussed, and absolute cross sections predicted.
\end{abstract}

PACS. 2 1.60.Jz,23.40.-s,26.30.-k

\section{Background for subject and title}

Almost two decades ago the paper entitled "The halo of the exotic nucleus ${ }^{11} \mathrm{Li}$ : a single Cooper pair" we (FB,RAB, EV and G. Colò) wrote with Pier Francesco Bortignon was published (Eur. Phys. J. A11, 385-392 (2001)). Since we started, few years before, thinking on this unstable, exotic nucleus, we became mesmerized by the possibilities the system offered, as a femtometer many-body laboratory to learn about the origin of pairing in nuclei. In particular, in connection with the E1-soft mode acting as the tailored glue of the two halo neutrons. ${ }^{1}$ Also to test the flexibility of nuclear field theory (NFT) to treat on

1 The paper had been completed a year before, passed the Editorial desk of Nature, and was rejected by the referee, himself a major figure in the field of nuclear structure, as I learned when he walked to me and referred, after my (RAB) presentation at the Conference "Bologna 2000- Structure of Nuclei at the Dawn of the Century", few months later, that the reason for the rejection had been the misunderstanding concerning our use or less of the bare $\mathrm{NN}^{1}{ }^{1} S_{0}$ pairing interaction. Interaction which is, in ${ }^{11} \mathrm{Li}$, subcritical to bind the two halo neutrons to the core ${ }^{9} \mathrm{Li}$. An outcome which Pier Francesco, who had dedicated much time and effort to peer review activity, being himself a superb practitioner of this difficult but necessary "art" could accept but not understand. He felt, in a deep sense, responsible of bringing the "truth" out of each paper he accepted to review. equal footing both (strongly and weakly) bound as well as continuum states. Furthermore to assess NFT abilities to lead to convergent results when simultaneously confronted with weak (induced interaction) and strong (selfenergy and parity inversion) particle-vibration coupling vertices 2 Our infatuation with the halo nuclei "laboratory" did not falter with the years, and the last paper we (the authors plus A. Idini) published with Pier Francesco on the subject, this time with the title "Unified description of structure and reactions: implementing the nuclear field theory", Phys. Scr. 91063012 (2016), presented an extension of NFT to simultaneously deal with structure and reactions, but also to spell in detail renormalization. Pier Francesco's efforts to connect these developments to well established results from the literature making use of his profound insight concerning the concepts and techniques of many-body physics, constituted a major inspiration. Pier Francesco's voice still rings in my (RAB) ears when he, after the task was completed, enthusiastically epitomised as "forte" the sentence introducing the corresponding discussion, namely "Enter empirical renormalization". Within this context it is only natural to dedicate the present paper to honour the memory of Pier Francesco, colleague and friend, reference point in finding the right path in our research.

\footnotetext{
2 A main contribution of Pier Francesco's lifelong work in NFT (see e.g. [1,2])
} 


\section{Introduction}

The nature of the (dipole) low-energy strength (LES) can be theoretically characterised by the response function (RF), the transition densities (TD) and the transition currents (TC). While $d B(E 1) / d E\left(\mathrm{e}^{2} \mathrm{fm}^{2} / \mathrm{MeV}\right)$ response function can be observed in e.g. $\left(\gamma, \gamma^{\prime}\right)$ experiments, the situation is less clear concerning TD and TC [3,4].

Experimentally, one has observed correlation existing between inelastic scattering reactions (ISR) and two-particle transfer reactions (TPTR) 5. The fact that ground state correlations (GSC) associated with a particle-hole $(p h)$ collective vibrations contribute constructively (destructively) coherent to the absolute ISR (TPTR) differential cross section while the opposite is true in the case of a $(p p)$ and $(h h)$ collective vibrations, can help at shedding light into the possible relations between the observable $B(E 1)$ response function, and the associated theoretical TD and TC of dipole LES. In particular in connection with the soft E1-mode of ${ }^{11} \mathrm{Li}$. The reasons for this selection are discussed in the next section.

\section{Nuclear embodiment of Cooper model and of a vortex}

Because the $N=6$ closed shell isotope ${ }_{3}^{9} \mathrm{Li}_{6}$ is a well bound system and ${ }_{3}^{10} \mathrm{Li}_{7}$ with one neutron outside closed shell is not, while ${ }^{11} \mathrm{Li}$ with two neutrons outside closed shells is bound, one can posit pairing to be at the basis of the binding of the two halo neutrons to the core ${ }^{9} \mathrm{Li}$. In keeping with the fact that a) the two-neutron separation energy of the latter system is $S_{2 n}=6.094 \mathrm{MeV}$, while that of ${ }^{11} \mathrm{Li}$ is $S_{2 n}=0.380 \mathrm{MeV}$ and, b) that the corresponding radii are $R=2.7 \mathrm{fm}$ and $R=4.6 \mathrm{fm}$, one can view the ground state of ${ }^{11} \mathrm{Li}$, considering the $p_{3 / 2}(\pi)$ odd proton as a spectator, to be the nuclear embodiment of a Cooper pair: two fermions moving in time reversal states (coupled to $J^{\pi}=0^{+}$), weakly interacting on top of a quiescent Fermi sea, leading to a very extended (halo), barely bound (quasi boson) system ([6], see also [7] $)^{3}$ (see Fig. 1] also Appendix A) . Specific, detailed support for this picture is provided by the two-neutron transfer process ${ }^{1} \mathrm{H}\left({ }^{11} \mathrm{Li},{ }^{9} \mathrm{Li}(\mathrm{gs})\right)^{3} \mathrm{H}$. Theory leads to a quantitative account of the experimental findings at the $10 \%$ level [10,11.

\footnotetext{
3 Removing one of the fermions the other becomes also unbound. Such a system is, in nuclei, an elementary mode of excitation namely a pair addition mode which in the case of ${ }^{11} \mathrm{Li}$ becomes a (halo) pair addition mode. Mode which is specifically probed by two-neutron (Cooper pair) transfer (tunnelling). Mode which can, in principle, be moved around and, arguably, found as the first excited $0^{+}$halo state of ${ }^{12} \mathrm{Be}$. That one dubs such pairing vibrational mode with transfer quantum number $\beta=+2$ a Borromean entity (see e.g. 8 and refs. therein), gives only one aspect of the physics at the basis of the BCS explanation of superconductivity, paradigm of spontaneous symmetry breaking theories, and of the prediction of the Josephson effect [9] which has provided relative voltage standards of 1 part in $10^{19}$.
}

Within this scenario the soft E1-mode (again viewing the odd $p_{3 / 2}(\pi)$ proton as a spectator), observed at $E_{x} \leq 1$ $\mathrm{MeV}$ with a width of $\Gamma \approx 0.5 \mathrm{MeV}$ and carrying $\approx 6-$ $8 \%$ of the TRK energy weighted sum rule [12,13], can be viewed as a $J^{\pi}=1^{-}$Cooper pair, that is a quantal nuclear vortex [14,15], namely the quantal structure which allows rotation around a symmetry axis.

The calculation of this soft E1 mode was carried out making use of Saxon-Woods potentials to approximately reproduce both protons and neutron single-particle states, and BCS to determine the associated occupation numbers. The continuum was discretised by placing the system in a spherical box of radius $R_{b o x}=40 \mathrm{fm}$, ensuring convergence. The QRPA solution of the full dipole response of ${ }^{11} \mathrm{Li}$ was determined making use of a separable dipole-dipole interaction of self-consistent strength in a two-quasiparticle basis with energies up to $50 \mathrm{MeV}$. The results lead to a soft mode of centroid $0.75 \mathrm{MeV}$ and FWHM $\Gamma=0.5 \mathrm{MeV}$, and carrying $6.2 \%$ of the EWSR, in overall agreement with the experimental findings. The associated $r^{2}$-weighted transition densities are typical of a dipole LES of light halo systems: out-of-phase motion of the neutron (skin) halo against a core in which protons and neutrons oscillate in phase [16] (Fig. 2) .

The calculations discussed above are adequate to describe the processes associated with the role of intermediate boson played by the two halo neutron to the core ${ }^{9} \mathrm{Li}$ (induced pairing interaction). Also to provide the basis to calculate the absolute differential cross sections and transition probabilities associated with the probing of the mode with inelastic scattering and particle transfer experiments (see Figs. 3 and 4 below).

However, if one would like to investigate the quantitative consequences of the interplay between particle-hole $(p h)$ - and particle-particle $(p p)$-like ground state correlations (GSC) one is forced to go beyond QRPA, and take into account the variety of renormalisation processes. Namely, self-energy of single-particle states and vertex corrections (see e.g. [7, 17, 18, 19, 20]). An ambitious, pluriannual project going beyond the framework of the present contribution.

Concerning the interplay between $(p p)$ - and $(p h)$-GSC, although seemingly new, this subject, namely the theoretical discussion and the experimental consequences of $p p$ and $p h$ contributions to nuclear vibrations has a long tradition. In particular in connection with $\beta$ - and pairingvibrations in superfluid quadrupole deformed nuclei, where they get mixed due to the spontaneous breaking of rotational invariance ${ }^{4}$. A subject started in the 1960's 21] and still very much open (see [22,23] and refs. therein). One knows of only few cases in which, due to a propitious distribution of single-particle levels around the Fermi energy, one can observe a clear signal, for example an important enhancement of the two-nucleon transfer absolute cross section typical of pairing vibrations around closed shell

\footnotetext{
${ }^{4}$ Similar to what happens in ${ }^{11} \mathrm{Li}$ between the dipole pair addition mode and the soft E1 mode, associated with a system poised to acquire a permanent dipole deformation (see App. B).
} 


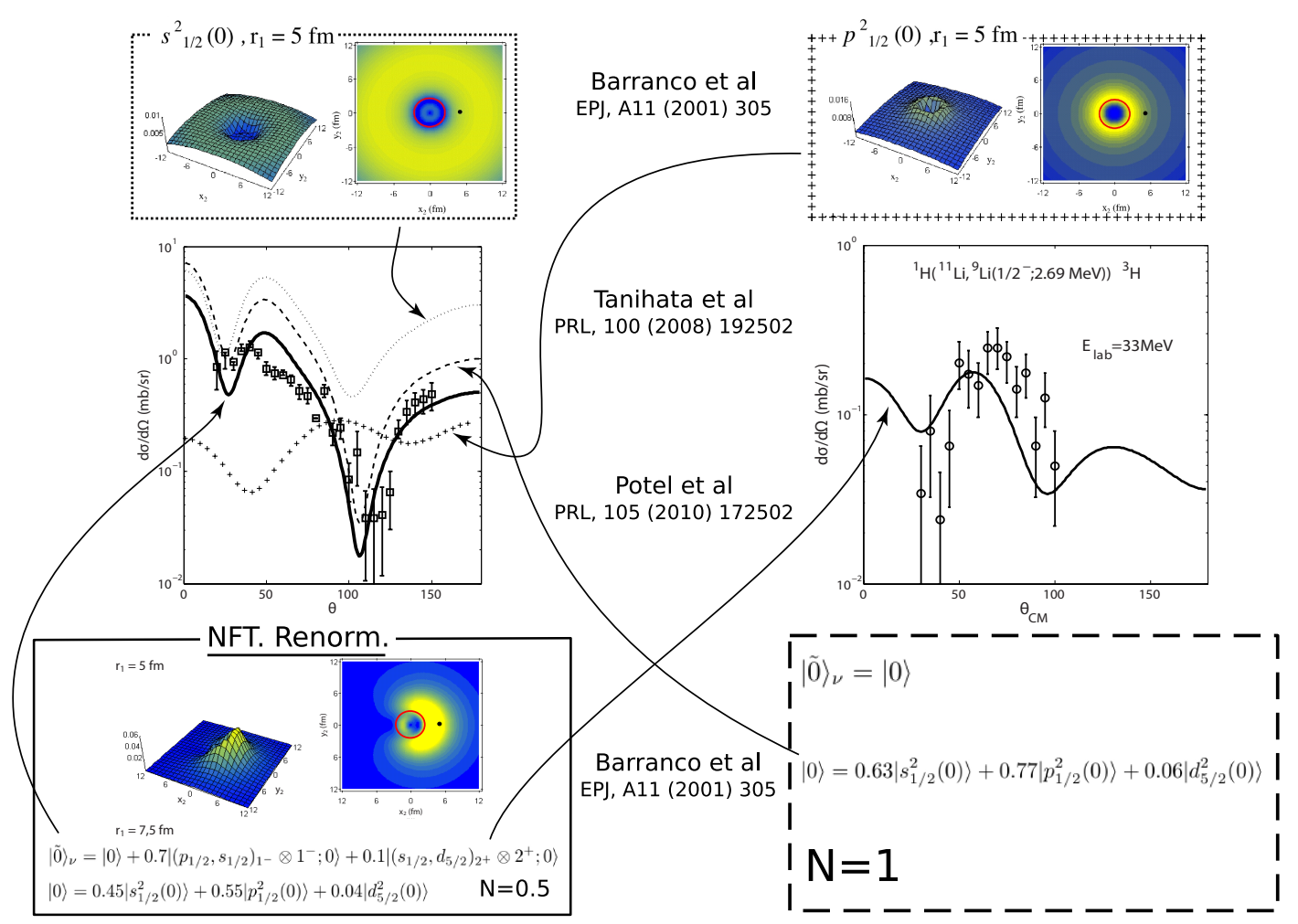

Fig. 1. Absolute, two-nucleon transfer differential cross section associated with the ground state and the first excited state of ${ }^{9} \mathrm{Li}$, excited in the reaction ${ }^{1} \mathrm{H}\left({ }^{11} \mathrm{Li},{ }^{9} \mathrm{Li}\right){ }^{3} \mathrm{H}$ in comparison with the predicted differential cross sections worked out making use of spectroscopic amplitudes and Cooper pair wavefunctions calculated with nuclear field theory.

systems rather than of superfluid nuclei (see [24, 25, 26 and refs. therein). Arguably, ${ }^{11} \mathrm{Li}$ constitutes such a propitious case concerning the characterisation of the dipole LES.

Let us close this section by reminding that the appearance of quantised vortices constitutes a hallmark of superfluidity. In a superfluid, a quantum vortex carries quantised orbital angular momentum, being zeros of the wave function around which the velocity field has a solenoidal shape. A nucleus acting as impurity immersed in a WignerSeitz cell of the inner crust of a neutron star (roughly equivalent to ${ }_{50}^{1000} \mathrm{Sn}$ ) experiences that a vortex becomes pinned by skating around it along the nuclear surface 14, [15]. This is in keeping with the fact that the sequence of levels of nuclei along the stability valley display, around the Fermi energy, a distribution of single-particle levels all carrying, exception made for the intruder one, the same parity. In the case of the neutron drip line nucleus ${ }^{11} \mathrm{Li}$, the outermost neutrons move with essentially equal amplitude $(\approx 0.7)$ in the almost degenerate $s_{1 / 2}$ and $p_{1 / 2}$ halo orbitals. Coupling the halo neutrons to angular momentum and parity $1^{-}$leads to a quantal vortex (Cooper pair with $J^{\pi}=1^{-}$) which again skates on the neutron (halo) skin. Said it differently, the soft $E 1$-mode of ${ }^{11} \mathrm{Li}$ can be viewed as a example of a quantum vortex in a nucleus ${ }^{5}$

\footnotetext{
5 This result provides also an answer to Nambu's last ques-
} tion in Section Conclusions and speculations of 27.
Let us now think in terms of a quantum superfluid. The ground state of ${ }^{11} \mathrm{Li}$ can be expressed as $\left|g s\left({ }^{11} \mathrm{Li}\right)\right\rangle=$ $\left|\Psi \otimes 1 p_{3 / 2}(\pi)\right\rangle$, where $\Psi=\sqrt{\alpha_{0}^{\prime}} e^{i \phi}$ parallels the GinzburgLandau wave function, $\phi$ the gauge angle and $|\Psi|^{2}=\alpha_{0}^{\prime}$ the superfluid density. In the present case it coincides with the modulus square of the halo neutron Cooper pair wavefunction 6 . Within this scenario the soft $E 1$-mode can be described in terms of the QRPA. It constitutes a twoquasiparticle, large amplitude mode, the estimated number of crossings being 7$] \approx 3$. We are thus confronted

\footnotetext{
${ }^{6}$ In other words, we are taking the first term of Eq. (A.1), namely the Cooper pair component expressed in terms of renormalised states $\left(\tilde{j}^{2}(0)\right)$ and amplitudes (note that the state in Eq. (A.2) is normalised to $\approx 1 / 2$ ). One could argue that a superfluid can hardly be made out of a single Cooper pair. This may be true but for this purpose one Cooper pair is hardly much worse than five Cooper pairs, the case of ${ }^{120} \mathrm{Sn}$, paradigm of superfluid nuclei. In any case, one can correctly view the ${ }^{11} \mathrm{Li}$ ground state as a pairing vibrational mode, vibrations which are very collective in nuclei, describe it in the RPA and construct with the corresponding $X$ and $Y$ amplitudes, the effective BCS $U_{\text {eff }}$ and $V_{\text {eff }}$ factors [28.

$7 n \approx(1 / 4 \sqrt{\pi}) \times A \times \beta([29,30]$ see also 31 Eq. (7.35)), with $\beta \approx r_{\text {extr }} / R\left({ }^{11} \mathrm{Li}\right) \approx 1.7$, the radius of ${ }^{11} \mathrm{Li}$ being $R\left({ }^{11} \mathrm{Li}\right)=$ $4.6 \mathrm{fm}$, while the extreme of the $r^{2}$-weighted halo transition density takes place at $r_{\text {extr }} \approx 8 \mathrm{fm}$ (Fig. 2).
} 
with a two-quasiparticle mode of angular momentum and parity $1^{-}$. Again the scenario of a quantal nuclear vortex.

\section{Two-nucleon transfer and detailed balance}

Our aim is that of calculating the absolute differential cross section associated with the reaction

$$
{ }^{9} L i+t \rightarrow{ }^{11} \operatorname{Li}\left(1^{-}\right)+p
$$

where the label $1^{-}$indicates states belonging to the soft E1-mode. But before doing so, we will proceed at assessing the accuracy with which such calculations can be carried out. This is in keeping with the fact that the prediction of $(\mathrm{t}, \mathrm{p})$ differential cross sections and the relative contribution to them of GSC can be of use to compare at profit with the results of active target and inverse kinematics experiments, to the extent that absolute cross sections can be worked out with sensible small errors, well below the $30 \%$ level. The experimental and theoretical absolute integrated cross sections associated with ${ }^{11} \mathrm{Li}(p, t){ }^{9} \mathrm{Li}(\mathrm{gs})$ for a bombarding energy of $3.3 \mathrm{MeV} / \mathrm{A}$ (Fig. 1), are $\sigma_{\text {exp }}(g s)=5.7 \pm 0.9 \mathrm{mb}\left[10\right.$ ] and $\sigma_{t h}(g s)=$ $6.1 \mathrm{mb}[11$ respectively, implying a deviation of the order of $7 \%$ (see also [32]). Let us now connect the $(\mathrm{p}, \mathrm{t})$ to $(\mathrm{t}, \mathrm{p})$ reaction through detailed balance, namely

$$
g_{\alpha} k_{\alpha}^{2} \frac{d \sigma}{d \Omega}(\alpha \rightarrow \beta)=g_{\beta} k_{\beta}^{2} \frac{d \sigma}{d \Omega}(\beta \rightarrow \alpha)
$$

where $8^{8} \alpha=\left({ }^{9} \operatorname{Li}(\mathrm{gs})+\mathrm{t}\right)$ and $\beta=\left({ }^{11} \mathrm{Li}(\mathrm{gs})+\mathrm{p}\right), k_{\alpha}, k_{\beta}$ and $g_{\alpha}, g_{\beta}$ being the relative linear momentum and the total number of spins in entrance and exit channel respectively. In the case under discussion $g_{\alpha}=g_{\beta}=3 / 2$ while $k_{\alpha}^{2}=7.425 \mathrm{fm}^{-2}$ and $k_{\beta}^{2}=0.72 \mathrm{fm}^{-2}$ resulting in $\sigma(\alpha \rightarrow \beta)=0.097 \times \sigma(\beta \rightarrow \alpha)=0.55 \pm 0.09 \mathrm{mb}$. As expected, the microscopic calculations carried out making use of the elements -wave functions and thus two-nucleon spectroscopic amplitudes, single-particle wave functions and optical potentials- and of the two-particle-transfer code COOPER [34 employed in [11] leads to the detailed balance result.

In what follows we calculate, for a triton bombarding energy of $15 \mathrm{MeV}$, the absolute differential cross section associated with the reaction (1) for the states associated with the soft E1-mode, examples of which are shown inf Fig. 3, as well as for the ground state transition ${ }^{9} \mathrm{Li}(t, p){ }^{11} \mathrm{Li}$ (gs) (Fig. 4(a)). Broadening each individual dipole state with a Lorentzian function and making use of the corresponding integrated cross section within the same angular range as that of the ground state transition $\left(20^{\circ} \leq \theta_{C M} \leq 154.5^{\circ}[10)\right.$, the two-neutron transfer dipole strength function $d \sigma\left(1^{-}\right) / d E(\mathrm{mb} / \mathrm{MeV})$ was constructed, and is displayed in Fig. 4(b). The subtended area (energy integration) is $\sigma\left(1^{-}\right)=0.13 \mathrm{mb}$, to be compared with $\sigma(g s)=0.51 \mathrm{mb}$. In all cases, the successive transfer of the two neutrons dominates over the simultaneous transfer (Fig. 4(a)). The reason being the very poor

\footnotetext{
${ }^{8}$ Concerning the notation see 33 .
}

overlap between the very extended single-particle neutron wave function involved in the structure of the soft E1mode and that associated with the triton. Within this context, we are technically in a two-center shell mode situation.

Similarly to $d \sigma\left(1^{-}\right) / d E$, making use of the transition densities associated with the dipole states, the dipole strength function $d B(E 1) / d E$ and associated Thomas-Reiche-Kuhn sum rule $(E d B(E 1) / d E)$ was calculated as a function of the energy. It is displayed in Fig. 4(c). The subtended area (energy integration) leads to $6.2 \%$ of the EWSR.

The similitude between the results displayed in Figs. 4(b) and 4(c) is apparent. The connection between theory and experiment (predictions which can be experimentally tested) are the two-nucleon transfer form factors in the first case, and the particle-hole transition densities in the second case. Ground state correlations affect quite differently (opposite) these theoretical quantities depending on whether they are of $(p h)$ or $(p p)$ type. Of particular interest are expected to be situations in which levels associated with the high (low) energy tail of the PDR (GDR) are close by in energy, so as to be able to probe on equal footing and with both TNTR and ISR large amplitude isoscalar modes with substantial $p p$ components, with small amplitudes isovector vibrations of mainly $p h$ character.

Summing up, we are in presence of a collective mode peaked at $E_{x} \leq 1 \mathrm{MeV}$, displaying a transition density consistent with that of a PDR, carrying a non-negligible fraction of the EWSR and an absolute two-neutron transfer cross section of the order of $25 \%$ of the ground state one. A laboratory to test, once the full renormalized nuclear field theory structure results become available, the texture of the associated ground state correlations through inelastic and electromagnetic processes, as well as twoparticle transfer ones, processes which are specific to shed light in the $(p h)$ and $(p p)$ aspects of the correlations respectively.

\section{Conclusions}

The combination of inelastic processes and of two-particle transfer reactions (strength functions) can be used at profit in characterizing the dipole LES provided one is able to calculate (TNTR) absolute differential cross sections at the $10 \%$ level accuracy, so as to be able to assess the role played by ground state correlations within experimental error. Such requirement may also imply, as in the present case, the calculation of absolute differential cross sections to continuum states.

A number of problems remain open, in particular concerning the fact that the wavelength of $\left(\gamma, \gamma^{\prime}\right)$ exciting the dipole LES is much larger than nuclear dimensions, let alone the fact that two-particle transfer is dominated by successive transfer, and that the associated form factor receives important contributions from configurations in which the two neutrons are essentially a nuclear diameter apart. This, together with the presence of substantial neutron excess leading to conspicuous isospin mixing, sets 


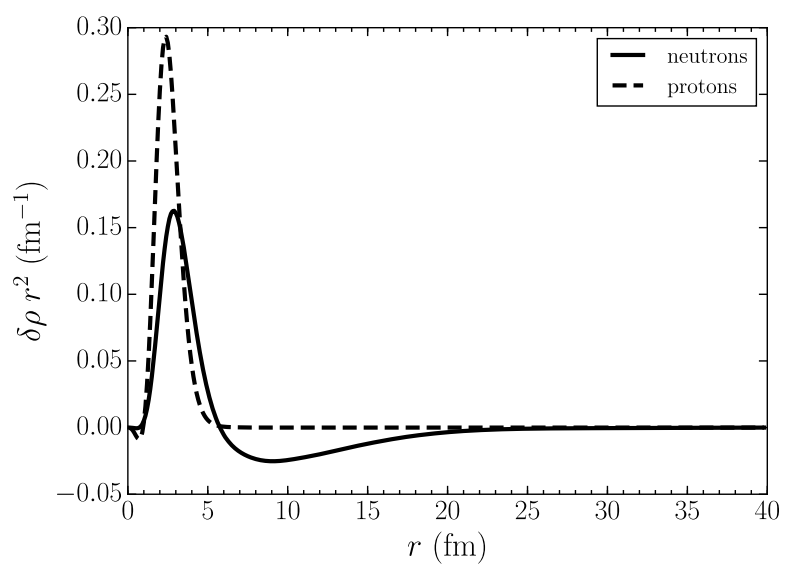

Fig. 2. Transition density multiplied by $r^{2}$ associated with states representative of the soft dipole mode of ${ }^{11} \mathrm{Li}$.
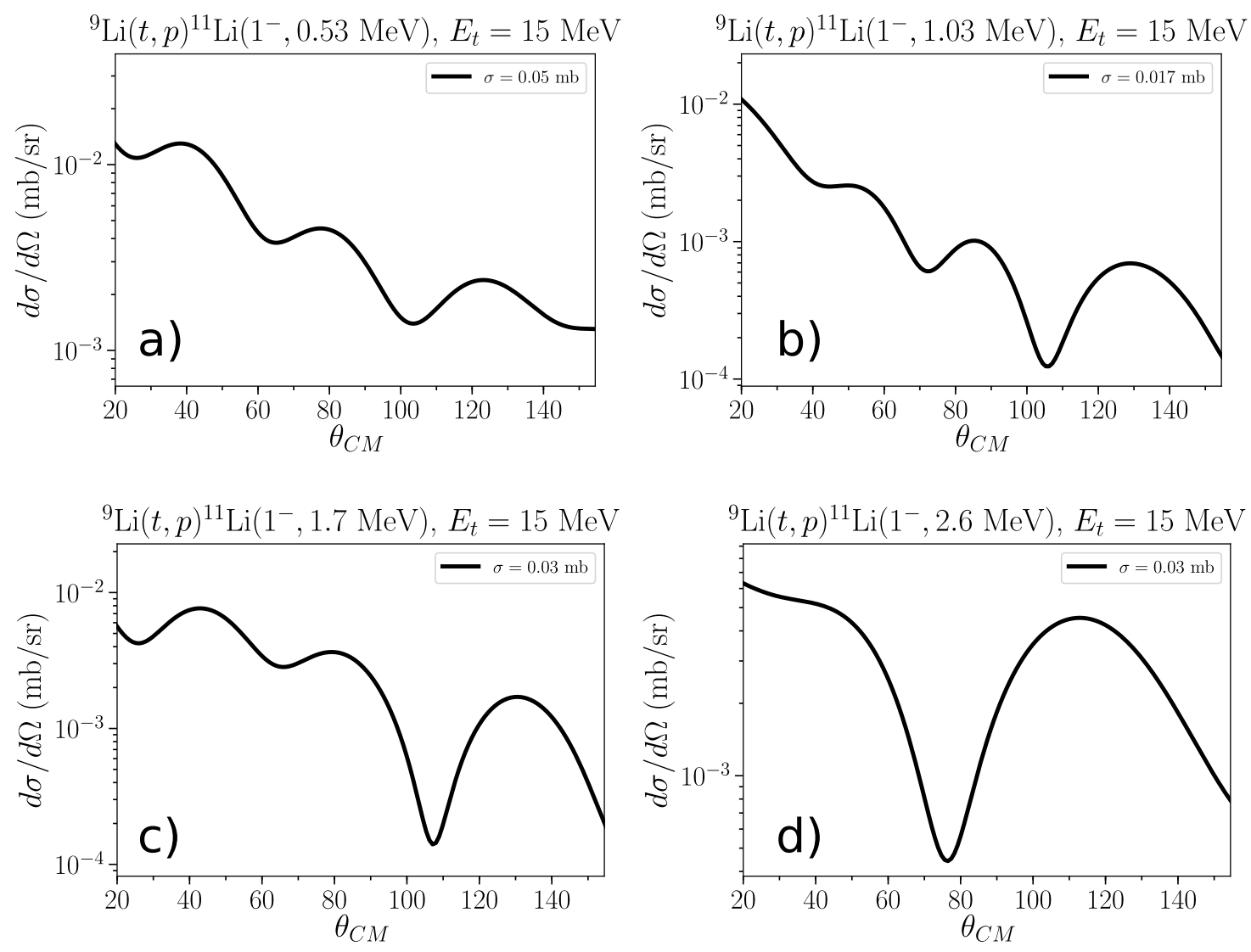

Fig. 3. Absolute two-nucleon differential cross section associated with states representative of the soft dipole mode of ${ }^{11} \mathrm{Li}$. The total absolute cross section results from the integration of $d \sigma / d \Omega$ in the angular interval $\left(20^{\circ} \leq \theta_{C M} \leq 154.5^{\circ}[10]\right.$.

a question mark on the "observability" of clear cut, distinct transition densities and of velocity fields. To which extent the measurement of magnetic moments could help at shedding light on some of these questions, remains both an experimental and theoretical open problem useful to look at.

\section{A Analytic calculation of ${ }^{11} \mathrm{Li}(\mathrm{p}, \mathrm{t})^{9} \mathrm{Li}(\mathrm{gs})$}

The ${ }^{11} \mathrm{Li}$ ground state wave function can be written, assuming the odd proton to act as a spectator, as $\left|g s\left({ }^{11} \mathrm{Li}\right)\right\rangle=\left|p_{3 / 2}(\pi)\right\rangle|\tilde{0}\rangle_{\nu}$ where,

$$
\begin{gathered}
\left.|\tilde{0}\rangle_{\nu}=|0\rangle+\mid \text { ind }\right\rangle, \\
|0\rangle_{\nu}=0.45\left|\widetilde{s}_{1 / 2}^{2}(0)\right\rangle+0.55\left|\widetilde{p}_{1 / 2}^{2}(0)\right\rangle+0.04\left|\widetilde{d}_{5 / 2}^{2}(0)\right\rangle,
\end{gathered}
$$



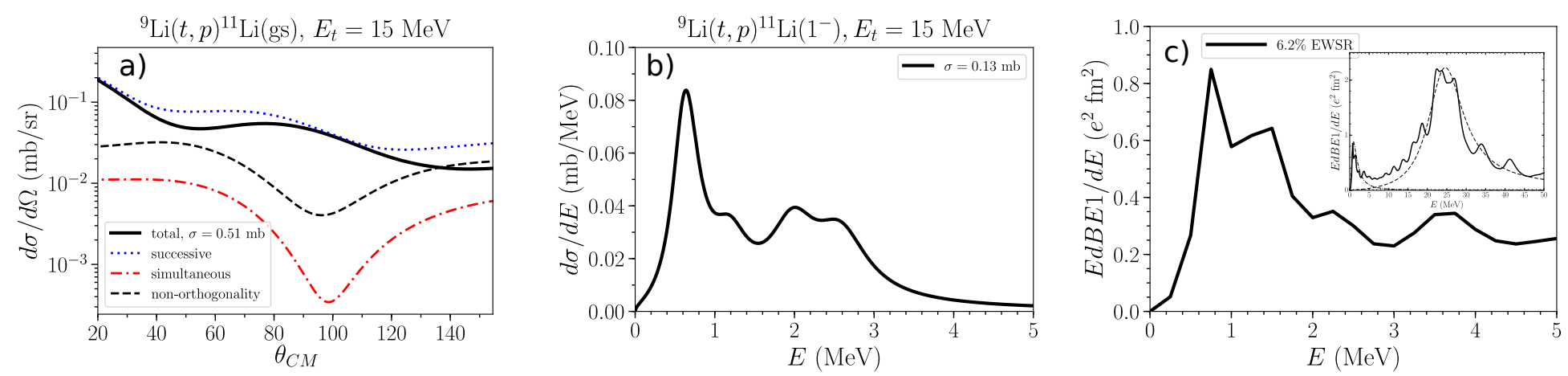

Fig. 4. (a) Absolute differential cross section associated with the ground state transition. The total absolute cross sections are calculated as explained in the caption to Fig. 3. (b) two-neutron transfer cross dipole strength function $d \sigma\left(1^{-}\right) / d E$, (c) EWSR $E d B E 1 / d E$ as a function of the energy.

and

$$
\mid \text { ind }\rangle=0.7\left|\left(\widetilde{s}_{1 / 2}, \widetilde{p}_{1 / 2}\right)_{1^{-}} ; 0^{+}\right\rangle+0.1\left|\left(\widetilde{s}_{1 / 2}, \widetilde{d}_{5 / 2}\right)_{2^{+}} ; 0^{+}\right\rangle,
$$

$\widetilde{s}_{1 / 2}, \widetilde{p}_{1 / 2}$ and $\widetilde{d}_{5 / 2}$ being renormalised single-particle states of ${ }^{10} \mathrm{Li}$ the first two lying at threshold with energies $\widetilde{\epsilon}_{\widetilde{s}_{1 / 2}}$ $=0.2 \mathrm{MeV}, \widetilde{\epsilon}_{\widetilde{p}_{1 / 2}}=0.5 \mathrm{MeV}$ [19]. Making use of the associated renormalised single-particle wave function [7, the two-nucleon transfer formfactor associated with the reaction ${ }^{1} \mathrm{H}\left({ }^{11} \mathrm{Li},{ }^{9} \mathrm{Li}(\mathrm{gs})\right)^{3} \mathrm{H}$ was worked out and the absolute differential cross section calculated [11. It provides a quantitative account of the absolute differential cross section and of the integrated experimental one [10]: $\sigma_{\exp }(g s)=$ $5.7 \pm 0.9 \mathrm{mb}, \sigma_{t h}(g s)=6.1 \mathrm{mb}$.

Let us now use the essentially two-component wave function (A.2) (see Fig. 5), to calculate this cross section in a simple fashion. Making use of Woods-Saxon singleparticle wavefunctions as formfactors the absolute differential cross section of the pure configurations $s_{1 / 2}^{2}(0)$ and $p_{1 / 2}^{2}(0)$ were calculated. The integrated values are

$$
\begin{gathered}
\sigma\left(s_{1 / 2}^{2}(0)\right)=23.17 \mathrm{mb}, \\
\sigma\left(p_{1 / 2}^{2}(0)\right)=2.16 \mathrm{mb} .
\end{gathered}
$$

Combining Eqs. (A.2)-(A.5) one can estimate,

$$
\sigma(g s)=(0.45 \sqrt{23.17}+0.55 \sqrt{2.16})^{2} \mathrm{mb}=8.86 \mathrm{mb},
$$

an estimate $45 \%((8.86-6.1) / 6.1)$ in error in relation to $\sigma_{t h}(g s)$.

At the basis of this discrepancy one finds the fact that the values of the cross sections reported in Eqs. (A.4) and (A.5) were calculated with bare and not renormalised formfactors. A simple estimate of the modifications of these formfactors due to renormalisation and associated couplings is related to the fact that $s_{1 / 2}$ experiences no centrifugal barrier as compared to the $p_{1 / 2}$ wavefunction. Because concentrated single-particle wavefunctions lead to larger matrix elements, and thus to cross sections, than less localised ones (think of the difference between neutron and proton pairing matrix elements), the use of renormalised formfactors will reduce the cross section displayed in Eq. (A.4) with respect to that shown in Eq. (A.5). A simple estimate can be made in terms of an effective diffusivity (for ${ }^{11} \mathrm{Li}$ ),

$$
a_{e f f}=\frac{R\left({ }^{11} \mathrm{Li}\right)}{R_{0}\left({ }^{11} \mathrm{Li}\right)} \times a=\frac{4.6}{2.7} \times 0.65 \mathrm{fm} \approx 1.1 \mathrm{fm},
$$

where $\mathrm{R}\left({ }^{11} \mathrm{Li}\right)=4.58 \pm 0.13 \mathrm{fm}$ is the measured radius of ${ }^{11} \mathrm{Li}$, while $R_{0}\left({ }^{11} \mathrm{Li}\right)=1.2 \times(11)^{1 / 3} \mathrm{fm}=2.7 \mathrm{fm}$. Assuming the $p_{1 / 2}$ single-particle wave function feels an effective potential of radius $R_{0}\left({ }^{11} \mathrm{Li}\right)+a_{\text {eff }}=3.8 \mathrm{fm}$, while the $s_{1 / 2}$ experience that of radius $R_{0}\left({ }^{11} \mathrm{Li}\right)+2 \times a_{e f f}=4.9$ $\mathrm{fm}$, one can estimate the relative decrease of the cross section (A.4) with respect to (A.5), i.e.: $\left(\frac{3.8}{4.9}\right)^{2} \times 23.17 \mathrm{mb} \approx$ $14 \mathrm{mb}$. The simple estimate (A.6) leads, in this case, to

$$
\sigma(g s)=(0.45 \sqrt{14}+0.55 \sqrt{2.16})^{2} \mathrm{mb} \approx 6.2 \mathrm{mb},
$$

a quantity which deviates by $\approx 1 \%((6.2-6.1) / 6.1)$ from $\sigma_{t h}(g s)$. In other words, a proper estimate of the cross sections shown in Eqs. (A.4) and (A.5) should be carried out making use of the renormalised radial wave functions $\widetilde{R}_{s_{1 / 2}}$ and $\widetilde{R}_{p_{1 / 2}}$.

The large error to be associated with the analysis of the experimental cross sections in terms of simple theoretical estimates making use of Woods-Saxon formfactors is rather suggestive. While one would not attempt a quantitative analysis of the spectrum of nuclei in terms of Woods-Saxon single-particle energies such an approach is still common praxis in attempting at extracting spectroscopic factors from transfer data. The deep interweaving existing between structure and reactions implies that structure amplitudes and reaction formfactors should be calculated at the same level of accuracy (renormalization) to be able to compare at profit theory with experiments (see e.g. [20]).

\section{B \\ (Spontaneously broken) spin-orbit symmetry, tensor force}

The essence of BCS pairing mechanism is Cooper pair formation due to a phonon-induced attraction [35,36]. It 
leads to a non zero pair correlation function, $\left\langle\sum_{\nu} a_{\nu}^{\dagger} a_{\tilde{\nu}}^{\dagger}\right\rangle=$ $\alpha_{0}^{\prime} e^{-2 i \phi}$, and thus to dynamical (spontaneous) symmetry breaking in gauge space. The magnitude of the associated deformation is measured by the number of Cooper pairs $\alpha_{0}^{\prime}$ which, divided by the appropriate volume leads to the pair density. It defines a privileged orientation in gauge space subtending a gauge angle $\phi$ with respect to the laboratory system of reference.

The chain of events "crystal formation" $\rightarrow$ "superconductivity" and associated violation of translational and gauge invariance, provides an example of the theoretical possibilities, tumbling in this case, related with spontaneously broken symmetry, in keeping with the fact that phonons are the Goldstone modes restoring Galilean invariance. In contrast, or more correctly, in parallel to the tumbling chain of symmetry breakings of descending energy scales, the idea of bootstrap is that the chain is circular and self sustaining [37.

The atomic nucleus provides a unique laboratory where the above concepts not only can be tested in terms of individual quantal states, as in the case of pairing rotational bands [39, 28, 40, but also in the limit of a single Cooper pair. In particular in the case of halo nuclei, much studied systems displaying large quantum fluctuations [41,42 whose interweaving can be described in physical and numerical detail, and tested in terms of absolute differential cross sections.

Within this scenario, a particularly attractive system is ${ }_{4}^{12} \mathrm{Be}_{8}$, with two neutrons outside the $\mathrm{N}=6$ closed shel ${ }^{9}$ This non-(Meyer-Jensen) magic number results from the phenomenon of parity inversion. Namely, from the quantal phase transition observed in ${ }^{11} \mathrm{Be}$ and resulting from the crossing of the $1 p_{1 / 2}$ and the $2 s_{1 / 2}$ levels and leading to the $1 / 2^{+}$ground state $\left(S_{n}=0.5 \mathrm{MeV}\right)$ and to the first excited $1 / 2^{-}$state $\left(E_{x}=0.32 \mathrm{MeV}\right)$. At the basis of these phenomenon one finds the self energy process controlled essentially by the coupling of the $1 p_{1 / 2}$ and the $2 s_{1 / 2}$ states with the low-lying quadrupole vibration of the core $\left(\hbar \omega_{2}=\right.$ $3.368 \mathrm{MeV}\left(\beta_{2} \approx 0.9\right)$ [43]). This implies a change from a regime in which static mean field effects dominate over quantal fluctuations, to another one in which the situation is essentially reversed. Saying it differently with the help of the definitions

$$
U(r)=\int d^{3} r^{\prime} \rho\left(r^{\prime}\right) v\left(\left|\boldsymbol{r}-\boldsymbol{r}^{\prime}\right|\right),
$$

and

$$
\delta U(r)=\int d^{3} r^{\prime} \delta \rho\left(r^{\prime}\right) v\left(\left|\boldsymbol{r}-\boldsymbol{r}^{\prime}\right|\right)
$$

from a situation in which $\bar{U} \gg \delta \bar{U}$, to one in which $\delta \bar{U} \geq$ $\bar{U}$. The function $U(r)$ is the Hartree potential while $\delta \bar{U}$ describes the quantal fluctuations of $U$ associated with vibrations of the system, in particular of the nuclear surface, while $\bar{U}=\left\langle\phi_{\nu_{F}}|U| \phi_{\nu_{F}}\right\rangle$ and similarly $\delta \bar{U}=\left\langle\phi_{\nu_{F}}|\delta U| \phi_{\nu_{F}}\right\rangle$,

\footnotetext{
${ }^{9}$ In this system, it is the first excited $0^{+}$state which can be viewed as a neutron halo Cooper pair, similar to the di-neutron component entering the ${ }^{11} \mathrm{Li}$ ground state (see footnote 3 ).
}

$\phi_{\nu_{F}}$ describing the single-particle motion of a nucleon at the Fermi energy.

Writing the Hamiltonian as $H=H_{0}+\beta_{2} H^{\prime}\left(\left[H_{0}, H^{\prime}\right]=\right.$ $0)$, where $H_{0}=T+U$ and with $H^{\prime}=\left(\frac{1}{16 \pi}\right)^{1 / 2}\left\langle R_{0} \partial U / \partial r\right\rangle$ (see [4], Eq. (6-68)) one observes that, starting from the $\mathrm{N}=7$ isotope ${ }_{6}^{13} \mathrm{C}$ displaying the standard $1 p_{1 / 2}-2 s_{1 / 2}$ sequence, and removing one proton at a time, the (quadrupole) particle-vibration coupling strength changes leading eventually to a crossing of the $1 / 2^{-}, 1 / 2^{+}$levels, this last becoming ground state for ${ }^{11} \mathrm{Be}$ (Fig. 6). Parity inversion also found in ${ }^{10} \mathrm{Li}([19]$ and refs. therein) 10

Being these systems at zero temperature, we are in presence of a quantal phase transition. Phase transition which induces the tumbling chain parity-inversion $\rightarrow$ presence of (dynamical) dipole moment in the ground state of ${ }^{11} \mathrm{Li}$. More accurately, the emergence of a soft E1-mode carrying about $6 \%$ of the TRK sum rule. Tumbling which, in the case of ${ }^{11} \mathrm{Li}$, becomes a bootstrap circular chain. In fact, the soft E1-mode of ${ }^{11} \mathrm{Li}$, which consists in a vibrational motion of the halo neutrons against the protons and neutrons of the core which vibrate in phase, acts as the glue of the two halo neutrons to the core ${ }^{9} \mathrm{Li}$ and thus to the very existence of the halo.

Given the success of BCS theory in the case of superconductivity, it was natural to ask whether a similar mechanism may also work for the fermionic superfluid ${ }_{2}^{3} \mathrm{He}_{1}$. In this case the attraction between the atoms must be an intrinsic property of ${ }^{3} \mathrm{He}$. The main feature of the inter-atomic potential is the strong repulsive component at short distances, and the weak Van der Waals attraction at medium and large distances. To avoid repulsion the ${ }^{3} \mathrm{He}$ atoms have to form Cooper pairs in a state of relative angular momentum $L$ different from zero, thus being kept away by the centrifugal barrier. It turned out that $L=1$ and $S=1$ 48.

Early experimental data indicated that the entities forming the Cooper pairs were not the bare ${ }^{3} \mathrm{He}$ atoms, but strongly dressed fermions, displaying effective masses about six times larger than the bare atomic mass ${ }^{11}$. It is

10 While the bare energies of the single-particle states are not observable, the $B(E 2)$ transitions probabilities $\left(\beta_{2}^{2}\right)$ of the lowlying collective states are so. The microscopic mechanism at the basis of parity inversion in ${ }^{11} \mathrm{Be}$ and ${ }^{10} \mathrm{Li}$ is parallel to that at the basis of the Lamb shift in the hydrogen atom $(\mathrm{H})$. Namely self energy and Pauli principle (see [45, Fig. 14.2, 43. Figs. 2(I)(a) and 2(II)(b)). In (renormalised) QED the bare electron mass is the (divergent) quantity one has to subtract so that the renormalised energy splitting between the ${ }^{2} S_{1 / 2}$ and ${ }^{2} P_{1 / 2}$ levels of the $\mathrm{H}$ atom best reproduces the experimental findings (1057.845(9) MHz (Exp.), $1057.865 \mathrm{MHz}$ ). In (renormalised) nuclear field theory, the (four) parameters of the bare Saxon-Woods potential plus $k$-mass are adjusted so as the renormalized single-particle energies best reproduce the observed quantities (see Fig. 6). Within this context it is of notice the remarkable prediction of parity inversion made in 46], and the detailed, profound analysis of the phenomenon presented in 47.

11 In the case of ${ }^{11} \mathrm{Li}$, the mass increase from the bare $(4 / 2$ $\mathrm{MeV}^{-1}, 4$ being the summed degeneracy of the $p_{1 / 2}$ and $s_{1 / 2}$ 
(a)<smiles>CC(C)C(C(C)C)C(C(C)C)C(C)C</smiles>

(b)

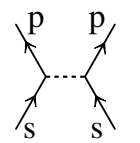

(c)

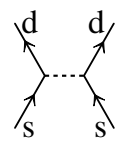

(d)<smiles>[2H]C(C)C(C([2H])C)C(C)(C)P</smiles>

(e)<smiles>CC(C)C(C)C(C)C(=P)I</smiles>

(f)<smiles>CC(C)C(C)C(C)C</smiles>

(g)<smiles>[2H]C(C)C(C)C(C)C(C)C(C)[C](C)C(C)C</smiles>

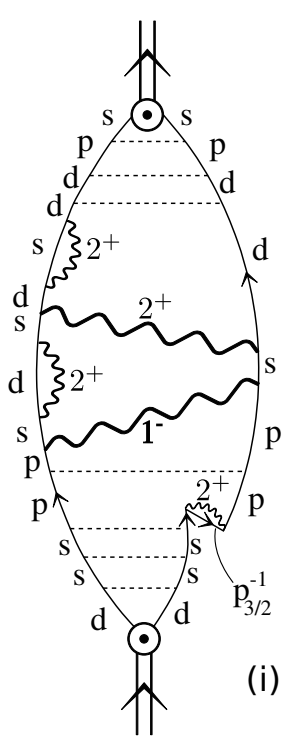

$$
\begin{aligned}
& \mathrm{s} \rightarrow \mathrm{s}_{1 / 2} \\
& \mathrm{p} \rightarrow \mathrm{p}_{1 / 2} \\
& \mathrm{~d} \rightarrow \mathrm{d}_{5 / 2}
\end{aligned}
$$

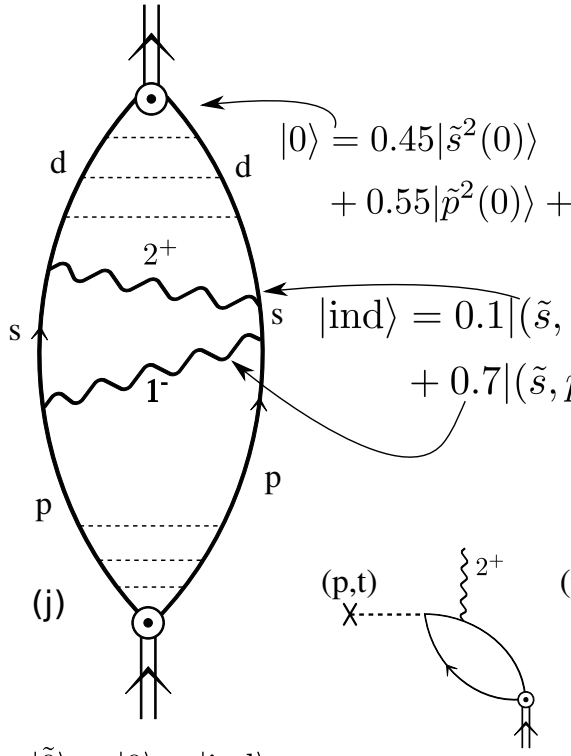

(k)



(I)

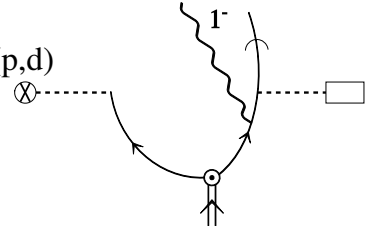

(m)

Fig. 5. Microscopic processes contributing to the structure of $\left|\tilde{0}_{\nu}\right\rangle$ (Eq. (A.1)). (a)-(f): bare ${ }^{1} S_{0}$ NN-interaction processes contribution to the correlation of the two-halo neutrons of ${ }^{11} \mathrm{Li}$ and to its binding to the core ${ }^{9} \mathrm{Li}$ leading to a contribution of the order of $-100 \mathrm{keV} ;(\mathrm{g}),(\mathrm{h})$ induced pairing interaction associated with the exchange of the low-lying quadrupole vibration of the core and of the soft E1-mode. This last one contributes with most of the two halo neutron separation energy $\left(S_{2 n} \approx 380 \mathrm{keV}\right)$; (i) nuclear field theory diagram associated with the renormalization of the single-particle states, and with their binding to the ${ }^{9} \mathrm{Li}$ core. Bold wavy lines describe dressed vibrational modes whose properties (energy and electromagnetic transition probabilities) reproduce the experimental findings (renormalised NFT); $(\mathrm{j})$ the connection between the diagrammatic processes and the Dirac (ket) representation (Eqs. (A.1)-(A.3)); (k) through the invasive, irreversible ${ }^{1} \mathrm{H}\left({ }^{11} \mathrm{Li},{ }^{9} \mathrm{Li}\left(1 / 2^{-} ; 2.769 \mathrm{MeV}\right)\right)^{3} \mathrm{H}$ process populating the $1 / 2^{-}$member of the $\left(2^{+} \otimes p_{3 / 2}(\pi)\right)_{1 / 2^{-}, \ldots 7 / 2^{-}}$multiplet of ${ }^{9} \mathrm{Li}$, evidence for the component $\left|(\tilde{s}, \tilde{d})_{2^{+}} \times 2^{+} ; 0^{+}\right\rangle$ of $\left|\tilde{0}_{\nu}\right\rangle$ was obtained [1] (Fig 1, see also [38]). Similar information can be gathered by recording the $\gamma-$ ray associated with the decay of the quadrupole mode of ${ }^{9} \mathrm{Li}$ in coincidence with the outgoing particle $\left({ }^{9} \mathrm{Li}\right)$, an experiment which remains to date of the gedanken type. However, it does not need to remain such, being a possible (and likely important) experiment, in keeping with the fact that the ${ }^{11} \mathrm{Li}$ beam had a rather low energy $(3.3 \mathrm{MeV} / A)$, the flying time to the particle detector after the reaction has taken place being adequate as compared to $\hbar / \Gamma_{\gamma}\left(\Gamma_{\gamma} \sim \mathrm{eV}\right)$, the main proviso to be made concerns the solid angle covered by the $\gamma$-detector; (l) in principle, the above inverse kinematics, two-neutron pickup process can provide similar information concerning the (symbiotic, bootstrap) $\left|(\tilde{s}, \tilde{p})_{1-} \otimes 1^{-} ; 0^{+}\right\rangle$component. However, in this case, the flying path is replaced by the interaction range $(\approx 10 \mathrm{fm})$, in keeping with the fact that the soft dipole mode is a vibration which involves the neutron (halo) skin which the reaction annihilates. Because the interaction time is 6-7 orders of magnitude shorter than than $\hbar / \Gamma_{\gamma}$, the process discussed will likely remain a gedanken experiment; $(\mathrm{m})$ similar as before but for the case of a single neutron pickup reaction leading to ${ }^{10} \mathrm{Li}$ which, not being bound, will loose the second neutron through coupling to the continuum (horizontal dashed line ending in an open square), the asymptotic wave describing its motion being represented by a curved arrow.

thus not surprising that the bare atomic potential bears little resemblance to the effective potential acting between the dressed fermions (quasiparticles). The most important consequence of the onset of Cooper pairing in liquid ${ }^{3} \mathrm{He}$ is the ability to amplify ultra weak effects, in particular those

states, $2 \mathrm{MeV}$ their energy separation) to the dressed one $\left(4 / 0.4 \mathrm{MeV}^{-1}\right)$ is of a factor of 5 , in keeping with the fact that the density of levels around the Fermi energy is inversely proportional to the effective mass. of the electromagnetic interaction between the nuclear dipole moments. Even at the distance of closest approach of two ${ }^{3} \mathrm{He}$ atoms (the hard-core radius $\mathrm{R} \approx 2 \AA$ ) this interaction is only of the order of $10^{-7} K$, orders of magnitude smaller than the critical temperature $\left(\approx 10^{-3} \mathrm{~K}\right)$. The amplification arises due to the fact that the system mediating the induced interaction (the quasiparticles) is 

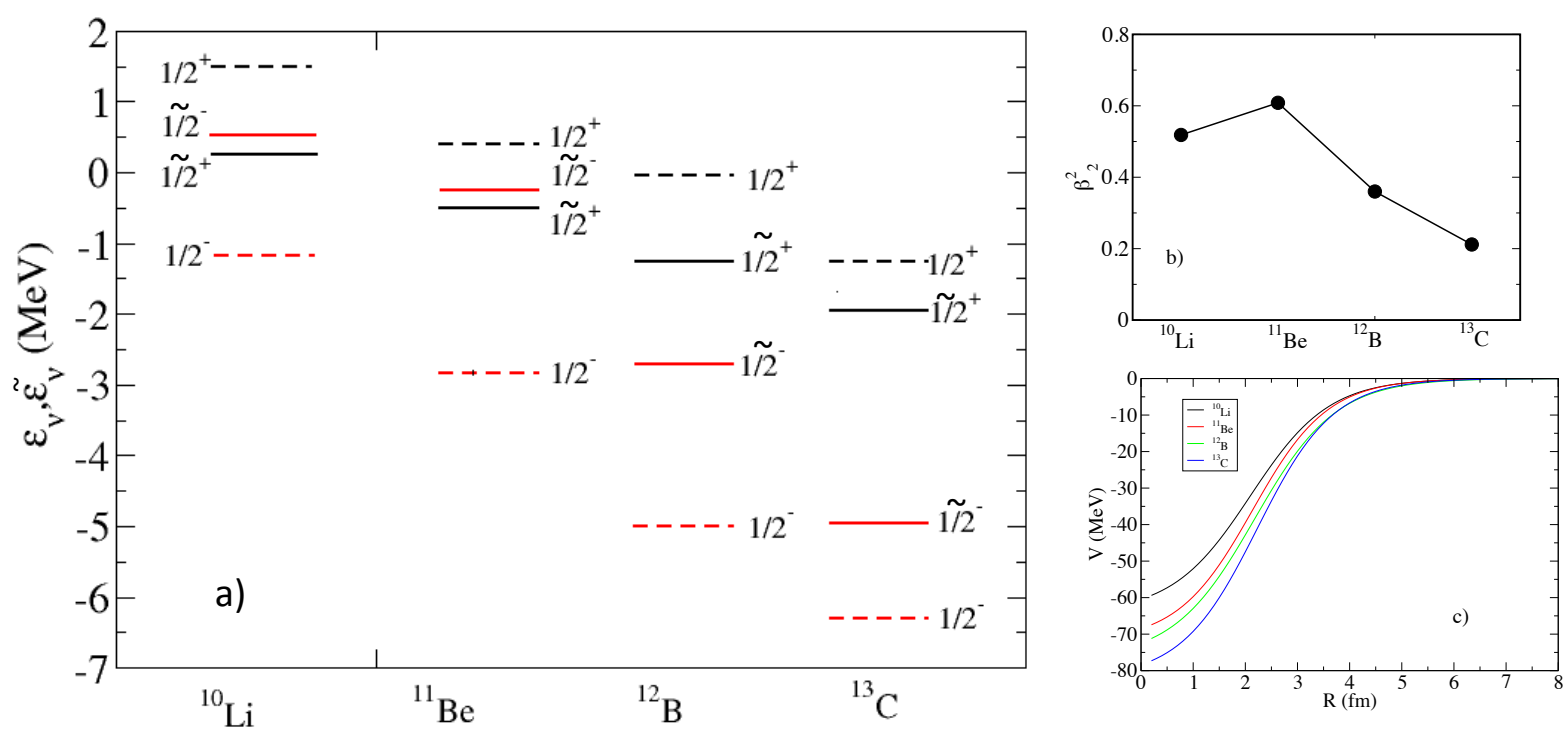

Fig. 6. (color online) Bare $\left(\epsilon_{\nu}\right.$, horizontal dashed) and renormalised ( $\tilde{\epsilon}_{\nu}$, horizontal continuous bold face) single-particle energies associated with the $1 / 2^{+}$(black lines) and $1 / 2^{-}$(red lines) states of the $N=7$ isotones. (b) The square of the dynamical deformation $\beta_{2}$ associated with the lowest quadrupole vibration of these nuclei are indicated with a solid dot. (c) The bare mean field potentials.

the very same system between which the interaction operates (bootstrap) 12 .

It is of notice that while the direct interaction between the nuclear dipole moments of two ${ }^{3} \mathrm{He}$ atoms is invariant under simultaneous rotation of $\boldsymbol{L}$ and $\boldsymbol{S}$, it is not invariant under rotation of either alone. Consequently, if the "strong" (Van der Waals, etc.) force in the problem forces all pairs to have e.g. a fixed and identical relation of their orbital orientation to their spin, the condensate will display a spontaneously broken spin-orbit symmetry (SBSOS) [49. That is, the symmetries with respect to rotation in spin and orbital space are spontaneously broken, leading to a highly degenerate ground state. In this situation, a tiny dipole-dipole interaction, which implies a spin-orbit coupling, is able to lift the degeneracy, by choosing that particular relative orientation of $\boldsymbol{L}$ and $\boldsymbol{S}$ for which the dipolar energy is minimal and in this case of the order of $N_{p} \times 10^{-7} \mathrm{~K}\left(N_{p} \approx 10^{22}\right.$, number of Cooper pairs). Thereby the interaction between the nuclear dipole moments acquires macroscopic relevance 50.

Central nuclear forces, in which the line of action passes through the pair of nucleons although their magnitude may depend on the relative orientation of their spins conserve $\boldsymbol{S}$ and $\boldsymbol{L}$ separately, as well as the total angular momentum $\boldsymbol{J}$ and parity $\pi$. The tensor force $V(r)=$ $V_{T}(r)\left\{3\left(\boldsymbol{\sigma}_{1} \cdot \hat{r}\right)\left(\boldsymbol{\sigma}_{2} \cdot \hat{r}\right)-\boldsymbol{\sigma}_{1} \cdot \boldsymbol{\sigma}_{2}\right\}$, where $\sigma_{1}$ and $\sigma_{2}$ are the Pauli matrices of the two nucleons, $\hat{r}$ is the unit vector parallel to the line joining them and $V_{T}(r)$ is some scalar

\footnotetext{
${ }^{12}$ As stated above, in ${ }^{11} \mathrm{Li}$ the system mediating the interaction between the two halo neutrons (the halo field) is the very same system through which the induced interaction operates (exchange of soft E1-mode between the halo neutrons).
}

function of their separation, is non-central and thus mixes states with the same $\boldsymbol{J}$ but different $\boldsymbol{L}$ and $\boldsymbol{S}$ [51.

While the tensor force is rather strong its influence on the properties of the nucleus still remains an open problem. This is to some extent due to the fact that its effects are mixed up with those of the spin-orbit force $V_{s o}(r) \boldsymbol{L} \cdot \boldsymbol{S}$.

To which extent the tumbling (bootstrap) chain quadrupole (dynamical) surface distortion $\rightarrow$ parity inversion (level crossing) $\rightarrow$ dipole (dynamical) distortion $\rightarrow$ (incipient) gauge invariance distortion at the single Cooper pair level found in the NFT description of ${ }^{11} \mathrm{Li}$ can be related to the tensor force [52,53] and, arguably, to some kind of SBSOS, is an open and challenging question.

\section{References}

1. Bortignon, P. F., R. A. Broglia, and D. R. Bès. On the convergence of nuclear field theory perturbation expansion for strongly anharmonic systems. Phys. Lett. B, 76:153, 1978.

2. P. F. Bortignon. Nuclear field theory of two-phonon states. In A. Bohr and R. A. Broglia, editors, International School of Physics "Enrico Fermi" Course LXIX, Elementary Modes of Excitation in Nuclei, page 519, Amsterdam, 1977. North Holland.

3. A. Repko, P. G. Reinhard, V. O. Nesterenko, and J. Kvasil. Toroidal nature of the low-energy E1 mode. Phys. Rev. C, 87:04305, 2013.

4. N. Ryezayeva, T. Hartmann, Y. Kalmykov, H. Lenske, P. von Neumann-Cosel, V. Yu. Ponomarev, A. Richter, A. Shevchenko, S. Volz, and J. Wambach. Nature of lowenergy dipole strength in nuclei: The case of a resonance at particle threshold in ${ }^{208} \mathrm{~Pb}$. Phys. Rev. Lett., 89:272502, 2002 . 
5. R. A. Broglia, C. Riedel, and T. Udagawa. Coherence properties of two-neutron transfer reactions and their relation to inelastic scattering. Nuclear Physics A, 169:225, 1971.

6. L. N. Cooper. Bound Electron Pairs in a Degenerate Fermi Gas. Phys. Rev., 104:1189, 1956.

7. Barranco, F., P. F. Bortignon, R. A. Broglia, G. Colò, and E. Vigezzi. The halo of the exotic nucleus ${ }^{11} \mathrm{Li}$ : a single Cooper pair. Europ. Phys. J. A, 11:385, 2001.

8. M.V. Zhukov, B.V. Danilin, D.V. Fedorov, J.M. Bang, I.J. Thompson, and J.S. Vaagen. Bound state properties of Borromean halo nuclei: ${ }^{6} \mathrm{He}$ and ${ }^{11} \mathrm{Li}$. Physics Reports, 231(4):151 - 199, 1993.

9. B. D. Josephson. Possible new effects in superconductive tunnelling. Phys. Lett., 1:251, 1962.

10. Tanihata, I., M. Alcorta, D. Bandyopadhyay, R. Bieri, L. Buchmann, B. Davids, N. Galinski, D. Howell, W. Mills, S. Mythili, R. Openshaw, E. Padilla-Rodal, G. Ruprecht, G. Sheffer, A. C. Shotter, M. Trinczek, P. Walden, H. Savajols, T. Roger, M. Caamano, W. Mittig, P. RousselChomaz, R. Kanungo, A. Gallant, M. Notani, G. Savard, and I. J. Thompson. Measurement of the two-halo neutron transfer reaction ${ }^{1} \mathrm{H}\left({ }^{11} \mathrm{Li},{ }^{9} \mathrm{Li}\right){ }^{3} \mathrm{H}$ at $3 \mathrm{~A} \mathrm{MeV}$. Phys. Rev. Lett., 100:192502, 2008.

11. G. Potel, F. Barranco, E. Vigezzi, and R. A. Broglia. Evidence for phonon mediated pairing interaction in the halo of the nucleus ${ }^{11}$ Li. Phys. Rev. Lett., 105:172502, 2010.

12. R. Kanungo, A. Sanetullaev, J. Tanaka, S. Ishimoto, G. Hagen, T. Myo, T. Suzuki, C. Andreoiu, P. Bender, A. A. Chen, B. Davids, J. Fallis, J. P. Fortin, N. Galinski, A. T. Gallant, P. E. Garrett, G. Hackman, B. Hadinia, G. Jansen, M. Keefe, R. Krücken, J. Lighthall, E. McNeice, D. Miller, T. Otsuka, J. Purcell, J. S. Randhawa, T. Roger, A. Rojas, H. Savajols, A. Shotter, I. Tanihata, I. J. Thompson, C. Unsworth, P. Voss, and Z. Wang. Evidence of soft dipole resonance in ${ }^{11} \mathrm{Li}$ with isoscalar character. Phys. Rev. Lett., 114:192502, 2015.

13. T. Nakamura et al. Observation of Strong Low-Lying E1 Strength in the Two-Neutron Halo Nucleus ${ }^{11} \mathrm{Li}$. Phys. Rev. Lett., 96:252502, 2006.

14. P. Avogadro, F. Barranco, R. A. Broglia, and E. Vigezzi. Quantum calculation of vortices in the inner crust of neutron stars. Phys. Rev. C, 75:012805, 2007.

15. P. Avogadro, F. Barranco, R.A. Broglia, and E. Vigezzi. Vortex-nucleus interaction in the inner crust of neutron stars. Nuclear Physics A, 811(3):378, 2008.

16. R.A. Broglia, F. Barranco, A. Idini, G. Potel, and E. Vigezzi. Pygmy resonances: what's in a name? arXiv:18096.09409; Phys. Scr., in press, 2019.

17. H. Lenske, F. Hofmann, and C.M. Keil. Probing isospin dynamics in halo nuclei. Progress in Particle and Nuclear Physics, 46(1):187 - 196, 2001.

18. Orrigo, S. E. A. and H. Lenske. Pairing resonances and the continuum spectroscopy of ${ }^{10} \mathrm{Li}$. Phys. Lett. B, 677:214, 2009.

19. F. Barranco, G. Potel, E. Vigezzi, and R. A. Broglia. $d\left({ }^{9} \mathrm{Li}, p\right)$, specific probe of ${ }^{10} \mathrm{Li}$, paradigm of parityinverted, soft-dipole isotones with one neutron outside the $N=6$ closed shell. arXiv:1812.01761.

20. R. A. Broglia, P. F. Bortignon, F. Barranco, E. Vigezzi, A. Idini, and G. Potel. Unified description of structure and reactions: implementing the Nuclear Field Theory program. Phys. Scr., 91:063012, 2016.
21. D. R. Bès. Beta-vibrations in even nuclei. Nuclear Physics, 49:544 - 565, 1963.

22. A. Aprahamian, R. C. de Haan, S. R. Lesher, C. Casarella, A. Stratman, H. G. Börner, H. Lehmann, M. Jentschel, and A. M. Bruce. Lifetime measurements in ${ }^{156} \mathrm{Gd}$. Phys. Rev. C, 98:034303, 2018.

23. Sharpey-Schafer, J. F., Bark, R. A., Bvumbi, S. P., Dinoko, T. R. S., and Majola, S. N. T. "stiff" deformed nuclei, configuration dependent pairing and the $\beta$ and $\gamma$ degrees of freedom. Eur. Phys. J. A, 55(2):15, 2019.

24. J. V. Maher, J. R. Erskine, A. M. Friedman, J. P. Schiffer, and R. H. Siemssen. Unexpected strong pair correlations in excited $0^{+}$states of actinide nuclei. Phys. Rev. Lett., 25:302-306, 1970

25. R.F. Casten, E.R. Flynn, J.D. Garrett, O. Hansen, T.J. Mulligan, D.R. Bès, R.A. Broglia, and B. Nilsson. Search for $(t, p)$ transitions to excited $0^{+}$states in the actinide region. Physics Letters B, 40:333, 1972.

26. I. Ragnarsson and R. A. Broglia. Pairing isomers. Nuclear Physics A, 263:315, 1976.

27. Madhusree Mukerjee and Yoichiro Nambu. BCS and IBM. Annals of Physics, 191(1):143 - 162, 1989.

28. Potel, G., A. Idini, F. Barranco, E. Vigezzi, and R. A. Broglia. Quantitative study of coherent pairing modes with two-neutron transfer: Sn isotopes. Phys. Rev. C, 87:054321, 2013.

29. Barranco, F., R. A. Broglia, and G. F. Bertsch. Exotic radioactivity as a superfluid tunneling phenomenon. Phys. Rev. Lett., 60:507, 1988.

30. Barranco, F., G.F. Bertsch, R.A. Broglia, and E. Vigezzi. Large-amplitude motion in superfluid fermi droplets. $\mathrm{Nu}$ clear Physics A, 512:253, 1990.

31. Brink, D. and R. A. Broglia. Nuclear Superfluidity. Cambridge University Press, Cambridge, 2005.

32. Potel, G., A. Idini, F. Barranco, E. Vigezzi, and R. A. Broglia. Cooper pair transfer in nuclei. Rep. Prog. Phys., 76:106301, 2013.

33. R. A. Broglia and A. Winther. Heavy Ion Reactions. Westview Press, Boulder, CO., 2004.

34. G. Potel. COOPER, code for two-nucleon transfer reactions. (unpublished).

35. J. Bardeen, L. N. Cooper, and J. R. Schrieffer. Microscopic theory of superconductivity. Physical Review, 106:162, 1957.

36. J. Bardeen, L. N. Cooper, and J. R. Schrieffer. Theory of superconductivity. Physical Review, 108:1175, 1957.

37. Y. Nambu. Dynamical symmetry breaking. In T. Eguchi and K. Nishigama, editors, Broken Symmetry, Selected papers of Y. Nambu (1995), page 436. World Scientific, Singapore, 1991.

38. F. Barranco, G. Potel, E. Vigezzi, and R. A. Broglia. Radioactive beams and inverse kinematics: probing the quantal texture of the nuclear vacuum. arXiv:1904.02786, 2019.

39. Bès, D. R. and R. A. Broglia. Pairing vibrations. Nucl. Phys., 80:289, 1966.

40. Nobuo Hinohara and Witold Nazarewicz. Pairing NambuGoldstone Modes within Nuclear Density Functional Theory. Phys. Rev. Lett., 116:152502, 2016.

41. F. Barranco, R. A. Broglia, G. Potel, and E. Vigezzi. Core polarization and neutron halos. Journal of Physics: Conference Series, 527:012005, 2014.

42. R. A. Broglia, G. Potel, F. Barranco, and E. Vigezzi. Difference between stable and exotic nuclei: medium polarization effects. J. Phys. G, 37:064022, 2010. 
43. F. Barranco, G. Potel, R. A. Broglia, and E. Vigezzi. Structure and reactions of ${ }^{11} \mathrm{Be}$ : many-body basis for singleneutron halo. Phys. Rev. Lett., 119:082501, 2017.

44. Bohr, A. and B. R. Mottelson. Nuclear Structure, Vol.II. Benjamin, New York, 1975.

45. S. Weinberg. The Quantum Theory of Fields, volume 1. Cambridge University Press, Cambridge, 1996.

46. I. Talmi and I. Unna. Order of Levels in the Shell Model and Spin of Be ${ }^{11}$. Phys. Rev. Lett., 4:469, 1960.

47. H. Sagawa, B. A. Brown, and H. Esbensen. Parity inversion in the $\mathrm{N}=7$ isotones and the pairing blocking effect. Phys. Lett. B, 309:1, 1993.

48. D. Vollhardt and P. Wölfle. The superfluid phases of Helium 3. Taylor and Francis, London, 1990.

49. A.J. Leggett. Quantum Liquids. Oxford University Press, Oxford, 2006.

50. P. W. Anderson. Considerations on the flow of superfluid helium. Rev. Mod. Phys., 38:298-310, 1966.

51. D. M. Brink. Nuclear Forces. Pergamon Press, Oxford, 1955 .

52. Takaharu Otsuka, Toshio Suzuki, Rintaro Fujimoto, Hubert Grawe, and Yoshinori Akaishi. Evolution of nuclear shells due to the tensor force. Phys. Rev. Lett., 95:232502, 2005.

53. Takayuki Myo, Kiyoshi Katō, Hiroshi Toki, and Kiyomi Ikeda. Roles of tensor and pairing correlations on halo formation in ${ }^{11}$ Li. Phys. Rev. C, 76:024305, 2007. 